\title{
Novel Approaches to Fine-Tune Therapeutic Targeting of Platelets in Atherosclerosis: A Critical Appraisal
}

\author{
Thorsten Kessler ${ }^{1,2}$ Heribert Schunkert ${ }^{1,2}$ Philipp von Hundelshausen ${ }^{2,30}$ \\ ${ }^{1}$ Deutsches Herzzentrum München, Klinik für Herz- und \\ Kreislauferkrankungen, Technische Universität München, \\ Munich, Germany \\ 2 Deutsches Zentrum für Herz-Kreislauf-Forschung (DZHK) e.V., \\ Partner Site Munich Heart Alliance, Munich, Germany \\ 3 Institut für Prophylaxe und Epidemiologie der Kreislaufkrankheiten, \\ Klinikum der Universität, Ludwig-Maximilians-Universität, Partner \\ Site Munich Heart Alliance, Munich, Germany \begin{abstract}
Germany (e-mail: thorsten.kessler@tum.de).
Philipp von Hundelshausen, MD, Institute for Cardiovascular Pettenkoferstraße 9, 80336 Munich, Germany (e-mail: philipp.von_hundelshausen@med.uni-muenchen.de).
\end{abstract} \\ Address for correspondence Thorsten Kessler, MD, Deutsches \\ Herzzentrum München, Klinik für Herz- und Kreislauferkrankungen, \\ Technische Universität München, Lazarettstraße 36, 80636 Munich, \\ Prevention, Ludwig-Maximilians-University (LMU) Munich,
}

Thromb Haemost 2020;120:1492-1504.

\begin{abstract}
Keywords

- platelets

- acquired

- atherogenesis

- lipids

- adhesion molecules

- inflammation

The pathogenesis of atherosclerotic vascular disease is driven by a multitude of risk factors intertwining metabolic and inflammatory pathways. Increasing knowledge about platelet biology sheds light on how platelets take part in these processes from early to later stages of plaque development. Recent insights from experimental studies and mouse models substantiate platelets as initiators and amplifiers in atherogenic leukocyte recruitment. These studies are complemented by results from genetics studies shedding light on novel molecular mechanisms which provide an interesting prospect as novel targets. For instance, experimental studies provide further details how platelet-decorated von Willebrand factor tethered to activated endothelial cells plays a role in atherogenic monocyte recruitment. Novel aspects of platelets as atherogenic inductors of neutrophil extracellular traps and particularities in signaling pathways such as cyclic guanosine monophosphate and the inhibitory adaptor molecule SHB23/LNK associating platelets with atherogenesis are shared. In summary, it was our intention to balance insights from recent experimental data that support a plausible role for platelets in atherogenesis against a paucity of clinical evidence needed to validate this concept in humans.
\end{abstract}

\section{Introduction}

We have experienced during the last decades a continuous progress in treating the complications of atherosclerosis such as myocardial infarction (MI), stroke, and acute limb ischemia by diagnostic improvements and continued development of new medical devices and drugs. Still, a large part of patients with acute ischemia does not manage in time to profit from these medical advances. ${ }^{1}$ Thus, the quest for novel targets aimed at a further individual reduction of the risk for a cardiovascular event by preventing atherosclerosis is justified. It has been argued that the sole causal risk factor for atherosclerosis is simply hypercholesterolemia and that other epidemiologically associated factors are either exacerbating or only bystander phenomena. ${ }^{2}$ It may be that by abolishing circulating low-density lipoprotein (LDL)-cholesterol atherogenesis might be completely preventable; whether such an approach is realistic remains questionable. In the meantime, it will be worthwhile to understand the mechanisms of atherogenesis to identify novel targets. Beyond the metabolic, there is received

January 13,2020

accepted after revision

June 10, 2020 (c) 2020 Georg Thieme Verlag KG Stuttgart · New York
DOI https://doi.org/

10.1055/s-0040-1714352. ISSN 0340-6245. 
an intricate chronic inflammatory component to consider. With the CANTOS trial, the hypothesis that cardiovascular events can be prevented by blocking a potent inflammatory target such as interleukin- $1 \beta$ has been proven. ${ }^{3}$ However, a clear cardiovascular benefit was outbalanced by the moderate effect size and fatal infections warranting further refinement through an individualized approach or testing new inflammatory targets. ${ }^{4}$ Especially $\mathrm{T}$ cells or monocytes, giving rise to intimal macrophages, but also almost every circulating blood cell type, have been described in plaques or to contribute to the inflammatory infiltrate and stability of the plaque. ${ }^{5}$ Irrespective of their pivotal role in arterial thrombosis, a causative role of platelets in atherogenesis has been suggested in the 1960s based on the concept that platelets represent a link between hemodynamic factors, lipids, and the characteristic localization of plaques. ${ }^{6}$ The concept of platelets as important inflammatory agents has been refreshed again in 2005 by Gawaz and colleagues explaining the proinflammatory machinery of platelets that intimately links thrombosis and atherosclerosis.? In recent years, rare genetic mutations with atherosclerosis phenotypes have been discovered and genome-wide association studies as well as Mendelian randomization studies have emerged as powerful tools generating big data that shed light and spark discussions about how platelets and blood cells may contribute to human atherosclerosis, defining novel targets for primary prevention.

\section{Platelets, Initiators, and Amplifiers of Atherogenic Leukocyte Recruitment}

Nearly our entire knowledge about the mechanisms of how platelets affect inflammation and atherosclerosis originates from animal models; preclinical data, however, have only partially been translated to the human system. The current understanding is that platelet activation is a requirement for their atherogenic properties. Hyperreactive platelets are associated with greater atherosclerotic plaque burden and increased plaque vulnerability, especially in culprit lesions in patients undergoing percutaneous coronary intervention (PCI) as measured by intravascular ultrasound (IVUS), and patients with more extensive coronary atherosclerosis have a higher number of hyperreactive platelets. ${ }^{8,9}$ It is thus possible that increased platelet reactivity may potentiate arterial thrombosis at the time of rupture, thereby driving inflammation and atherosclerotic lesion progression.

A crucial factor after platelet activation is the upregulation and activation of adhesion receptors that initiate and enhance the contact of platelets with (1) leukocytes to form aggregates and with (2) endothelial cells or their underlying matrix when exposed after injury. Key players are $\alpha I I b \beta 3$, P-selectin, the von Willebrand factor (VWF) receptor complex (GPIb $\alpha / \mathrm{V} / \mathrm{IX})$, and glycoprotein VI (GPVI). ${ }^{7}$ These adhesion molecules and their binding partners lead to an increase in rolling and firm adhesion of platelets on endothelial cells, and tether circulating leukocytes to the artery as a requirement for subsequent migration into the intima. ${ }^{10}$ Other adhesion molecules behave in a counterintuitive way: the selective genetic deficiency of JAM-A in platelets results in hyperreactive platelets and an increase in the formation of atherosclerotic lesions as JAM-A interacts with and inhibits $\alpha$ IIb $\beta 3$ activation, which also results in chemokine release ( $\mathbf{- F i g . ~ 1 ) . ~}{ }^{11}$ However, a celltype-dependent expression of JAM-A may be decisive whether JAM-A is atherogenic or atheroprotective. The expression of JAM-A on endothelial cells guides monocytes into flow-dependent predilection sites of atherosclerosis and JAM-A plasma levels are increased in coronary artery disease (CAD). ${ }^{12,13}$ Therefore, generalized JAM-A inhibition could turn out to be a two-sided sword.

Several functionally relevant chemokines are expressed and released by platelets. ${ }^{14}$ Chemokines tend to oligomerize which leads to the formation of mostly homodimers and heterodimers of either a CC-type (interaction of the $\mathrm{N}$-terminal part) or a CXC-type (extension of the $\beta$-sheet). This is important because atherogenic monocyte recruitment by CCL5, CXCL4, and their heterodimers depends also on these features. It can be therapeutically addressed by peptide inhibitors that are derived from amino acid sequences of the interface and protect from atherosclerosis. ${ }^{15,16}$

\section{GP1ba Interactions with von Willebrand Factor Mediate Platelet Adhesion and Promote Atherosclerosis}

Platelets have been described as initiators of atherosclerosis because they adhere to the arterial endothelium of the carotid artery in $\mathrm{ApoE}^{-1-}$ mice before atherosclerotic lesions become visible in a VWF- and GP1b-dependent process. ${ }^{17}$ VWF is stored and released upon injury or under inflammatory conditions from endothelial Weibel-Palade bodies or platelet $\alpha$-granules. It bridges collagen and activates a receptor complex upon multimerization (GPIb $\alpha / \mathrm{V} / \mathrm{IX})$, which is exclusively expressed by megakaryocytes and platelets leading to platelet adhesion on endothelial cells and driving early as well as midstage atherosclerosis in mouse models. ${ }^{17}$ The presence of activated VWF on atherosclerosis-prone endothelium has been confirmed by molecular imaging detecting GP1b-conjugated microbubbles by ultrasound in vivo. ${ }^{18}$ Notably, the source of VWF affects its functionality, possibly by altered glycosylation such as reduced $\mathrm{N}$-terminal sialylation and reduced affinity for GPIb $\alpha .{ }^{19}$ This might explain why in mouse models only endothelial cell-derived VWF but not platelet-derived VWF promotes atherosclerosis. ${ }^{20}$

After binding and activation of endothelial CD 40 by CD40L, ultralarge VWF-platelet strings arise and facilitate monocyte diapedesis. ${ }^{21}$ In conjunction with a reduced activity of ADAMTS13, which cleaves ultralarge VWF, and consequently higher amounts of ultralarge VWF in plasma of patients with $\mathrm{CAD}$, this mechanism has been proposed to contribute to enhanced monocyte recruitment at atherosclerotic predilection sites. ${ }^{21}$ Elevated VWF levels in humans are strongly associated with an increased risk of ischemic cardiovascular events. Whether this relation is causal or whether increased VWF levels just reflect disturbances of endothelial function remains to be elucidated. ${ }^{22}$ It would be very interesting to translate these finding to human genetic disorders that are comparable to mouse models. Robust large-scale prospective 
A

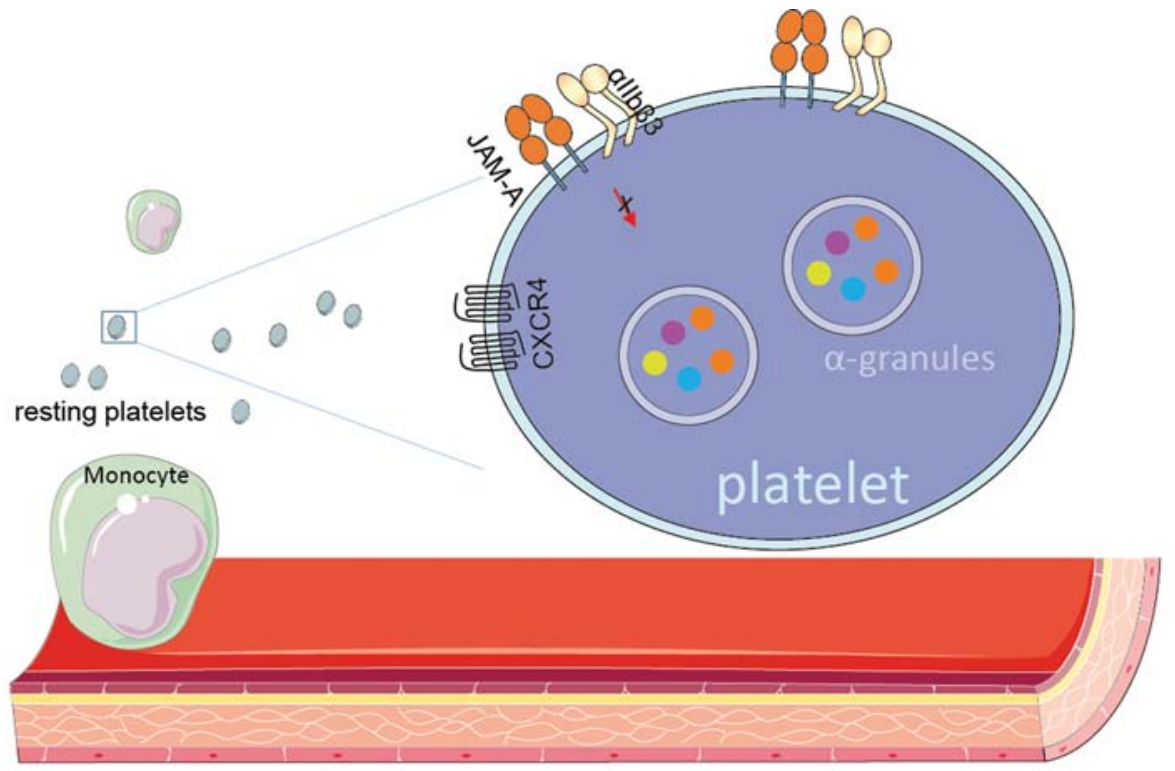

B

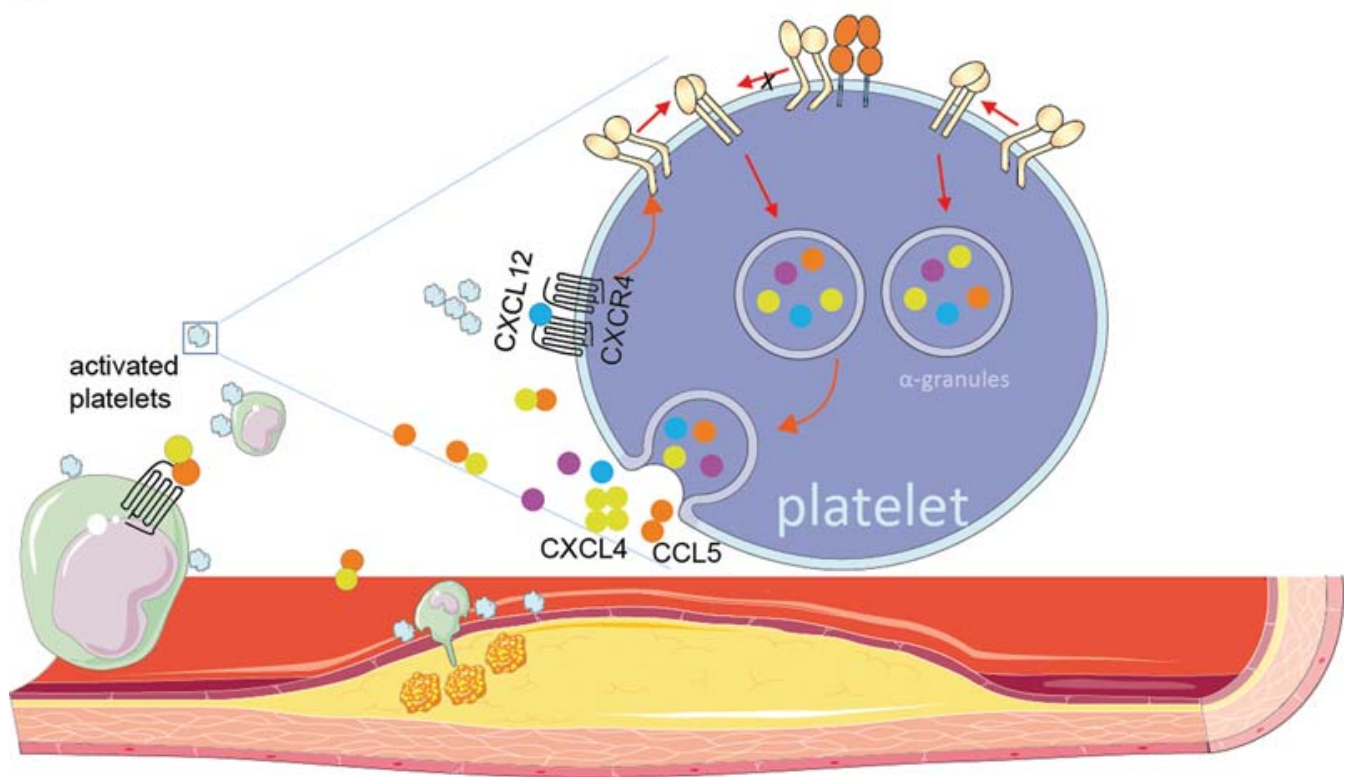

Fig. 1 Atheroprotective role of platelet JAM-A. The integrin $\alpha$ llb $\beta 3$ and JAM-A are coexpressed on platelets. Either by direct interaction or possibly via contacts through intermediary proteins lead the presence of JAM-A to reduced outside-in signaling of $\alpha$ llb $\beta 3$ (A). Genetic depletion of JAM-A from platelets results in increased outside-in signaling and consequently hyperreactive platelets (B). These hyperreactive platelets tend to form aggregates and complexes with monocytes and interact with dysfunctional endothelium to release chemokines such as CXCL4, CCL5, and CXCL12 which drives the generation of early atherosclerotic lesions.

clinical data that confirm a protection from atherosclerosis are, however, missing so far for both the rare Bernard-Soulier syndrome (GP1b $\alpha$ deficiency) and the common von Willebrand disease (VWD) where VWF is dysfunctional and levels are decreased. Lately, a Swedish registry of 2,790 individuals found that cardiovascular disease (CVD) mortality was more than halved in patients with VWD compared with controls while hospitalization due to a cardiovascular event was increased by $30 \% .{ }^{23}$ This might indicate an increase of rupture-prone lesions or lower stability of atherosclerotic lesions, whereas the lower mortality might be associated with a protection from arterial thrombosis. In aggregate, VWF appears to be a therapeutic target but experimental and clinical data are not consistent and require further investigation.

\section{P-selectin}

P-selectin is expressed on both endothelial cells and platelets. It is known for quite some time that the presence of P-selectin on both cellular entities can exacerbate atherosclerosis as shown by bone marrow transplantation models and adoptive transfer of P-selectin-deficient platelets. ${ }^{24,25}$ More recently, based on these findings and aiming to prevent the anti- 
inflammatory and antithrombotic platelet-dependent processes that are instrumental in atherogenesis, a P-selectin blocking monoclonal antibody (inclacumab) has been developed and tested in humans with NSTEMI, which resulted in decreased peri-interventional myocardial damage. ${ }^{26}$ As a side note, P-selectin expression is enhanced in platelets and endothelial cells in patients with sickle cell disease contributing to the risk of vaso-occlusive crises. A humanized monoclonal antibody, crizanlizumab, has been shown in the SUSTAIN trial to protect patients with sickle cell disease from vaso-occlusive crises and has recently been approved by the Food and Drug Administration for this indication. ${ }^{27}$

Although promising, randomized controlled trials over a longer period in a preventive setting and monitoring plaque development would be warranted to draw conclusions on beneficial effects of blocking P-selectin on atherogenesis in humans.

\section{Glycoprotein VI}

GPVI is one more target in atherogenesis. GPVI is a plateletspecific membrane protein that is primarily known for its interaction with fibrillar collagen but other ligands such as fibronectin or vitronectin are also known. ${ }^{28}$ These ligands are thought to be the binding partners for GPVI in atherogenesis since the subendothelial localization of collagen precludes their encounter at intact endothelium. ${ }^{29}$ Platelets adhere to the endothelium of early atherosclerotic arteries which can be diminished by antagonists like GPVI-Fc that can be coupled to microbubbles or by monoclonal antibodies, which goes hand in hand with a lesser extent of atherosclerosis. ${ }^{29,30}$ The inhibition of GPVI appears to be especially attractive as side effects are expected to be low since humans with a genetic deficiency of GPVI and respective knockout mice have only a mild bleeding diathesis. ${ }^{31} \mathrm{GPVI}-\mathrm{Fc}$ is envisioned to be powerful in preventing atherothrombosis without causing bleeding because collagen is an important component of atherosclerotic plaque activating platelets via GPVI and GPVI-Fc is most effective under high shear stress, but not low shear rates. ${ }^{32,33}$ GPVI-Fc (revacept) has entered clinical trials. A phase I trial showed that the drug was well tolerated and currently phase II trials are being performed to test its effectiveness in preventing periprocedural $\mathrm{PCI}$-associated events. ${ }^{34,35}$ The parenteral application of GPVI-Fc however hampers its use in primary prevention. An alternative option is orally available inhibitors of the Bruton's tyrosine kinase that interfere with the downstream signaling of GPVI and GPIb. ${ }^{36-38}$

\section{Platelet and NETs in Atherosclerosis: Guilty by Association}

Activities of platelets and neutrophils are closely intertwined and join forces in inflammation and atherosclerosis. Formation of neutrophil extracellular traps (NETs; NETosis) emerges as a potential important link between these cellular entities. ${ }^{39}$ Upon activation, neutrophils release decondensed chromatin decorated with granule proteins forming extracellular fibers that bind and kill bacteria. ${ }^{40}$ Critical for the unfolding of the chromatin structure is the enzyme peptidylarginine deiminase (PAD4) that catalyzes the citrullination of histones thereby uncoiling chromatin. ${ }^{41}$

The presence of NETs has been shown in sections of human atherosclerotic lesions, both at the luminal aspect and within murine atherosclerotic lesions. ${ }^{42,43} \mathrm{~A}$ role of NETs in propagating atherosclerosis is further supported by the finding that pharmacological interventions blocking NET formation via PAD4 inhibition can reduce atherosclerosis and arterial thrombosis in mice. ${ }^{44}$ Several mechanisms have been proposed to explain the proatherosclerotic role of NETs: e.g., neutrophilderived granule proteins (e.g., cathelicidin) stimulate a type I interferon response and cause endothelial dysfunction ${ }^{45}$ and cholesterol crystals induce NETs which prime macrophages for atherogenic IL- $1 \beta$ release. ${ }^{43}$ Furthermore, smooth muscle cells (SMCs) from atherosclerotic lesions attract neutrophils and trigger NETosis which in turn causes arterial tissue damage and inflammation. ${ }^{46}$

A role of platelets in NETosis was noted by observing that plasma from humans suffering from severe sepsis induced TLR4-dependent platelet-neutrophil interactions, leading to the production of NETs and clearing of bacteria. ${ }^{47}$ Under various conditions of activation, platelets have been demonstrated to trigger neutrophils to expel their NETs. In addition, platelet inhibitors proved to be protective by preventing NET formation in neutrophil-platelet-dependent diseases such as acute lung injury and atherosclerosis. ${ }^{39,48}$ Furthermore, in chronic inflammation platelet microparticles contribute to vasculopathy. This is fostered by their interaction with neutrophils and depends on the nuclear danger molecule HMGB1, which triggers neutrophils to cast their NETs. ${ }^{49,50}$

Platelets store and release chemokines that can form heteromers such as the chemokine CXCL4 that binds to CCL5 leading to synergistic effects on leukocyte recruitment and which can elicit NETosis in combination but not alone. ${ }^{51}$ Blocking this heterodimerization reduces atherosclerosis, lung injury, and the formation of NETs. ${ }^{51,52}$ Reciprocally, cell-free NETs induce platelet aggregation which depends on cathepsin $\mathrm{G}^{53}$

In summary, both activated platelets and NETs alone have been shown to play a role in experimental atherosclerosis. However, there is interdependency as activated platelets bind to and activate neutrophils which eventually leads to NETosis. $^{39,45,54}$ What is unknown so far is to what extent each component drives atherosclerosis independently. Although it is known that platelets induce NETs which then play a role in atherosclerosis, the concept that NETs represent a causative link between activated platelets and atherosclerosis has yet to be proven.

\section{Platelets and Lipids in Atherosclerosis: A Complex Relationship}

Based on the LIPID MAPS classification system, lipids can be classified into eight categories (fatty acids [FAs], glycerolipids, [glycerol-]phospholipids, sphingolipids, sterols, prenols, saccharolipids, and polyketines).$^{55}$ While all these are detectable in platelets, only few examples of prenols, saccharolipids, and 
polyketines have been detected and are therefore not further discussed in this section. ${ }^{56}$ Nevertheless, several prenols, saccharolipids, and polyketines have important functions in platelet biology and have been reviewed elsewhere. ${ }^{57-59}$

\section{Polyunsaturated Fatty Acids Are Regulators of Platelet Activity and Affect Atherosclerosis}

FAs can harbor multiple double bonds (PUFAs, polyunsaturated FAs). The position of the first double bond at the methyl end (omega, opposite the carboxyl group) explains the terminology so that omega-3 (n-3) FAs such as eicosapentaenoic acid (EPA) are differentiated from omega-6 FAs like arachidonic acid (AA). Integrated into phospholipids of the plasma membrane, FAs influence the fluidity and stability of cell membranes. Bulkier molecules such as the n-3 FA docosaheptaenoic acid result in greater membrane fluidity than FAs that fit better into the membrane geometry such as EPA. ${ }^{60}$ This may have important implications for the activity of platelets and other cell types and be one of various reasons why clinical trials in cardiovascular prevention using PUFA (consisting of mixtures) reported ambiguous results. Nevertheless, the REDUCE-IT trial, which corroborates the significant protective effects of pure EPA on cardiovascular events in the JELIS study, reignited the interest in using omega-3 acids in preventing atherosclerosis. ${ }^{61,62}$ Various preclinical data shed some light on the manifold, incompletely understood mechanisms, including reduced inflammation by effects on $\mathrm{T}$ cells and enhancement of resolution by lipid mediators, enhanced cholesterol efflux, antioxidant properties, and last but not least inhibitory effects on platelets. ${ }^{60} \mathrm{~A}$ hint that these effects on platelet activation might be clinically relevant comes from the tendency toward more bleeding events under EPA in REDUCE-IT. ${ }^{61}$ Feeding EPA to rabbits increases the incorporation of EPA into platelets and reduces collagen-induced platelet aggregation. ${ }^{63} \mathrm{FAs}$ are part of phospholipids in the platelet membrane and get released by cytoplasmic phospholipase A2 to serve as a substrate for platelet cyclooxygenase (COX-1), lipoxygenase (12-LOX), and CYP50 epoxygenases yielding various platelet inhibitors and activators. While AA serves as a precursor for the potent platelet activator thromboxane $\mathrm{A} 2$ and an opponent of the potent platelet inhibitor PGI2 (prostacyclin), EPA can be metabolized in platelets to various inhibitory lipids like thromboxane $A 3{ }^{59}$ These lipid mediators have a short half-life so that they are generated on demand. Adding AA to whole blood rapidly induces platelet activation in an autocrine manner via thromboxane binding to its $\mathrm{G}_{\mathrm{i}}$-coupled receptor TP on platelets, whereas PGI2 is produced by endothelial cells and activates in a paracrine fashion its $\mathrm{G}_{\mathrm{s}}$-coupled receptor IP. Deletion of TP results in diminished platelet reactivity and reduced atherosclerosis, whereas the knockout of IP accelerated atherosclerosis and decreased the stability of the lesions. Hence it appears that platelet activation through lipid mediators can be an important regulator of atherosclerosis. ${ }^{64-66}$ The platelet-specific effects of low-dose aspirin result from irreversible inhibition of COX-1 in platelets. Effects on PGI2 and thromboxane A2 are only the tip of the iceberg as low-dose aspirin in humans leads to drastic changes of the platelet FA profile and of other lipids that on top seem to vary considerably between donors. ${ }^{56}$
Aspirin is established in secondary but not in primary cardiovascular prevention. In humans low-dose aspirin completely abrogates platelet-derived thromboxane generation without reducing C-reactive protein (CRP) levels. ${ }^{67}$ Whether aspirin can reduce atherosclerosis in humans remains unclear; in light of many disappointing results in the setting of primary prevention, the latest being the ASPREE trial, large effects on atherogenesis seem unlikely. ${ }^{68} \mathrm{~A}$ failure of low-dose aspirin to reduce residual inflammation, as assessed with the most established prognostic risk marker in CVD, CRP, could be a possible explanation.

\section{Sphingolipids}

Sphingolipids are components of the cell membrane and regulate signaling. They comprise a group of molecules that are derivatives of ceramide which is mainly generated at the cytosolic side of the endoplasmic reticulum by assembling the amino acid serine and palmitoyl-CoA. Enzymatic reactions with ceramide as a substrate result in sphingosine and further steps produce sphingosine-1-phosphate (S1P), glucosylceramide, lactosylceramide, and sphingomyelin, which are important signaling molecules in inflammation and atherogenesis. $^{69-71}$ Analysis of atherosclerotic plaques revealed that sphingolipids are important plaque components and contribute to plaque inflammation and stability. ${ }^{72}$

Different to the on-demand metabolism of FA, platelets store S1P in granules and in nongranular compartments. ${ }^{73}$ Platelet activation leads to a release of S1P upon activation. As platelets express S1P receptors such as S1PR1, this results in a positive feedback mechanism. ${ }^{74}$ Moreover, expression of S1PR1 on megakaryocytes is required for normal thrombopoiesis as S1P drives cytoplasmic extensions of megakaryocytes into bone marrow sinusoids to shed proplatelets into the circulation. ${ }^{75}$ Although S1P is considered a proinflammatory mediator, platelet-derived S1P may have atheroprotective properties: it accelerates endothelial cell proliferation and drives endothelial cell migration. ${ }^{76}$ Sphingomyelin affects platelet reactivity through its incorporation into and formation of specialized regions within platelet membranes, lipid rafts, where important platelet adhesion receptors including GPVI and GPI-V-IX or scavenger receptors such as CD36 are embedded and that are required for a proper function. ${ }^{57}$ Another lipid species, phospholipids, is essential in these rafts fostering signaling through G-protein-coupled receptors by providing important second messengers. ${ }^{77}$

\section{(Glycero-)Phospholipids}

Phospholipids are the main component of cell membranes composed of glycerol derivatized by two hydrophobic acyl groups and a polar phosphate group. Depending on the molecules attached to the phosphate, phosphatidic acid (PA), phosphatidylcholine (PC), phosphatidylethanolamine $(\mathrm{PE})$, phosphatidylserine (PS), and the phosphatidylinositolrelated species (PI-PIP3) are classified. Cleavage of phospholipids by phospholipase $C$ generates phosphoinositide second messengers in signal transduction (e.g., PIP, $\mathrm{IP}_{3}$ ) and diacylglycerol, whereas lysophosphatidylcholine (LPC) arises after removal of an alkyl group of PC and a subsequent 
step by the phospholipase D autotaxin forms lysophosphatidic acid (LPA).

Under resting conditions the aminophospholipids PE and PS remain located at the inner leaflet of the platelet membrane. Platelet activation and apoptosis lead to $\mathrm{Ca}^{2+}$-dependent activation of a phospholipid transporter, the scramblase TMEM16F, that leads to the translocation of PS and PE to the outer leaflet thereby exposing binding sites for Annexin V and coagulation factors. ${ }^{78}$

Furthermore, the comparison of the platelet lipid profiles from CAD patients with matched controls reveals that LPC is weakly detectable in platelets of healthy persons, but increased by several orders of magnitude in CAD patients and also found in vulnerable atherosclerotic plaques. ${ }^{79,80}$ LPC has been reported to be concentrated in microvesicles from activated platelets which are important markers and factors in vascular inflammation and atherosclerosis. ${ }^{81}$ Lipid profiling of platelet MV identified differences in the composition with higher amounts of PC and LPC compared with the activated parent platelets. ${ }^{80}$ Platelets express the lysophospholipid receptor G2A/GPR132 that is responsible for platelet activation through LPC. $^{80}$

LPA, a derivative of LPC, is implicated in platelet activation and atherosclerosis signaling through G-protein-coupled receptors of the LPAR (EDG) family. LPA is produced by platelets through phospholipase A1 and autotaxin and is one active component of mildly oxidized LDL and atherosclerotic plaques in platelet activation. ${ }^{82,83}$

\section{Sterols/Cholesterol}

Hypercholesterolemia in humans correlates with platelet count.$^{84}$ As specified above, the relevance of the platelet count and platelet indices in humans for atherosclerosis remains vague/obscure. However, antibody-induced selective depletion of platelets inhibits atherogenesis significantly. ${ }^{85}$ Inversely, it is conceivable that an increased generation of platelets by hypercholesterolemia is atherogenic although the mechanisms how cholesterol levels modulate thrombopoiesis are unresolved. ${ }^{86}$ Reducing cholesterol and circulating LDL levels affects platelet reactivity by cholesterol-dependent and pleiotropic, cholesterol-independent effects. ${ }^{87}$ Clinically, pleiotropic, antithrombotic actions of statins have been concluded from the JUPITER trial that showed a significant reduction of deep vein thrombosis in the cohort treated with rosuvastatin. ${ }^{88}$ Which molecular mechanisms lie behind these assumed antiplatelet effects have been investigated in preclinical studies. Statins inhibit the synthesis of cholesterol by blocking HMG-CoA-reductase and as a side effect other lipids such as farnesyl pyrophosphate and geranylgeranyl pyrophosphate are missing for posttranslational prenylation of the small GTP-binding proteins Rho and Rac so that their activity is reduced. ${ }^{89}$ Rho and Rac are ubiquitously expressed and are indispensable regulators of the platelet cytoskeleton with various effects on downstream pathways including nitric oxide signaling. ${ }^{90}$ However, the size of the attributed pleiotropic effects on platelet reactivity in vivo is difficult to separate from the effects that occur as a result of the reduction of cholesterol: hypercholesterolemia alone increases platelet activation via binding of LDL and oxLDL to platelet
CD36. ${ }^{91,92}$ Novel treatment options for hypercholesterolemia as antibodies against PCSK9 also display antiplatelet characteristics: in a small clinical study the treatment with PCSK9 inhibitors resulted in a decrease of platelet P-selectin, platelet aggregation, and released proteins from $\alpha$-granules like CXCL4..$^{93}$ Levels of circulating PCSK9 correlate with platelet activity in acute coronary syndrome patients. ${ }^{94}$

\section{Genetic Evidence for a Role of Platelets in Coronary Artery Disease}

\section{Platelet Parameters}

Hematologic parameters including platelet count or mean platelet volume (MPV) have been associated with atherosclerosis and cardiovascular risk. ${ }^{95,96}$ Larger platelets have been described to be more reactive and to have a greater prothrombotic potential so that MPV has been found to be a useful prognostic parameter in MI. ${ }^{95}$ Although MPV is a readily available parameter with implications to platelet function, it is not standardized. Multiple factors including preanalytical issues such as time-dependent swelling of platelets in EDTA affect MPV so that using MPV is not a standard in clinical practice. ${ }^{97}$ As hematologic parameters show high interindividual variability, large genome-wide analyses of hematologic parameters have been undertaken to identify genetic variants that influence traits of red and white blood cell counts, but also platelet indices. - Supplementary Table S1 (available in the online version) gives an overview of studies reporting genetic associations with platelet phenotypes. In 2009 a first large systematic genome-wide meta-analysis ${ }^{98}$ identified 15 loci determining the MPV that jointly explained $8.6 \%$ of the total genetic variance in $\mathrm{MPV}$, but only to $0.5 \%$ of the platelet count. ${ }^{98}$ The most interesting region associated with platelet count and MPV was a haplotype restricted to Europeans located on chromosome 12q24 comprising 10 common single nucleotide polymorphisms (SNPs) including a nonsynonymous SNP Arg262Trp (rs3184504) in the gene SH2B3 associated with atherosclerosis and MI (see below). This haplotype is of importance because it significantly associates with premature CAD. ${ }^{99}$ In more recent and even larger studies searching for cardiometabolic risk factors in Europeans, more loci and variants have been discovered and refined that are more strongly associated with platelet count and explain the variance of platelet count to more than $8 \%{ }^{100}$ Signals for platelet count were mostly found within genes for congenital (GFI1B, THPO) or acquired $(A P O H)$ platelet disorders, underscoring that more subtle genetic variation within genes known to contain loss-of-function variants may reflect interindividual differences in these complex traits. ${ }^{100}$ In the last and so far most powerful study, more than 1,000 variants were identified to define platelet indices. In a Mendelian randomization study these variants displayed a weak, unexpectedly inverse relationship of coronary heart disease (CHD) and MPV suggesting shared causal pathways for $\mathrm{CHD}$ and MPV, although the mechanisms behind remain to be clarified. ${ }^{101}$

Taken together the results of these studies suggest that functional properties are more important for the role of platelets in atherosclerosis than the number of platelets 
(-Supplementary Table s1, available in the online version). ${ }^{101-108}$

As a prominent example, the cytochrome P450 2C19 genotype has been associated with a response to clopidogrel therapy. ${ }^{109,110}$ Genome-wide analyses also identified several genetic variants identified with platelet aggregation after stimulation with different agonists. ${ }^{111}$ Additionally, genes or pathways involved in platelet biology or function have also been identified to be associated with coronary atherosclerosis (for a review, see Erdmann et $\mathrm{al}^{112}$ ), e.g., the $S H 2 B 3$ gene and nitric oxide signaling.

\section{SH2B3/LNK-A Coronary Artery Disease Risk Gene with a} Role in Platelet Function

As stated above, the $S H 2 B 3$ gene is located on 12 q24 at a very complex genomic locus which shows associations with a variety of traits, e.g., type 1 diabetes, ${ }^{113}$ blood pressure, ${ }^{114,115}$ celiac disease, ${ }^{116}$ but also CAD $^{99}$ and platelet count. $^{98,101}$ The gene encodes LNK, an inhibitory adaptor protein regulating cytokine signaling and cell cycle in endothelial and hematopoietic cells. ${ }^{117,118}$ LNK prevents the signal transduction from a receptor tyrosine kinase to downstream JAK2, such as the signaling from TPO via MPL that triggers thrombopoiesis. Therefore, loss of LNK signaling results in thrombocytosis and increased platelet activation by $\alpha$ IIb $\beta 3$ outside-in signaling. ${ }^{117,119,120}$ In a study designed to explain the mechanism of the common CAD risk variant which results in a loss of function of LNK, Wang et al demonstrated that this variant causes an increase in platelet count especially under proatherogenic conditions, i.e., highcholesterol levels. L $^{-1-}$ mice displayed enhanced platelet activation, more leukocyte-platelet complexes, and accelerated arterial thrombosis and atherosclerosis. Specifically, hypercholesterolemia in $\mathrm{Lnk}^{-1-}$ mice led to enhanced interleukin-3/granulocyte-macrophage colony-stimulating factor receptor signaling but also increased platelet activation. ${ }^{121}$ In summary, platelet LNK is now an experimental and genetic link between, on the one side, high cholesterol levels, high platelet counts, high platelet reactivity and on the other side increased atherosclerotic plaque formation and MI. Enhancing LNK to inhibit platelet generation and activation might be an innovative strategy to reduce cardiovascular risk

\section{Multiple Genes Involved in Nitric Oxide Signaling}

Several genes which encode proteins that play a prominent role in nitric oxide signaling have been associated with CAD in genome-wide association studies (for a review see Wobst et $\mathrm{al}^{122}$ ): NOS3, which encodes the endothelial nitric oxide synthase (eNOS), ${ }^{123}$ GUCY1A1 (formerly named GUCY1A3), which encodes the $\alpha 1$-subunit of the soluble guanylyl cyclase (sGC), ${ }^{124}$ MRVI1, which encodes inositol 1,4,5-trisphosphate receptor-associated cyclic guanosine monophosphate (cGMP) kinase substrate (IRAG), ${ }^{125}$ and PDE5A, which encodes phosphodiesterase 5A (PDE5A), are the most prominent examples. However, several genes encoding proteins in related pathways, e.g., $P D E 3 A$, encoding phosphodiesterase $3 \mathrm{~A},{ }^{126}$ or the genes $E D N-1^{127,128}$ and EDNRA, ${ }^{124}$ encoding endothelin 1 and its receptor, respectively, have also been associated with CAD.

In the vasculature, nitric oxide is produced by eNOS mainly in endothelial cells leading to production of the second messenger cGMP in, e.g., vascular SMCs (VSMCs) and platelets by sGC. Accumulation of cGMP leads to relaxation of $\mathrm{VSMC}^{129}$ and inhibition of platelet aggregation, respectively. ${ }^{130,131}$ One mechanism is that the elevation of endogenous NO levels leads to reducing the thiol reductase activity of protein disulfide isomerase by S-nitrosylation which prevents platelet aggregation, $\alpha$-granule release, and thrombin generation on platelets. ${ }^{132}$ These NO effects, which also have been shown to influence, e.g., vascular remodeling ${ }^{133}$ or vascular inflammation, ${ }^{134,135}$ are limited by the breakdown of cGMP into GMP by PDE5A. Pharmacological modulation of these processes is used in a variety of diseases: supplementation of nitric oxide donors to relief angina pectoris, stimulators of SGC in pulmonary hypertension and heart failure, and PDE5A inhibitors in pulmonary hypertension and erectile dysfunction. MRVI1, the gene encoding IRAG, which represents a target of cGMP-dependent intracellular signaling, has also been associated with platelet aggregation. ${ }^{111}$ The variants at all of these loci, i.e., NOS3, GUCY1A1, PDE5A, and MRVI1, are located in noncoding regions. However, at least for NOS3, GUCY1A1, and PDE5A, an association between genotype and gene expression has been reported, i.e., the risk alleles of NOS3 and GUCY1A1 lead to reduced gene expression. ${ }^{136-138}$ As a consequence cGMP availability is reduced whereas the PDE5A risk allele is associated with increased gene expression. ${ }^{139,140}$ While the effect at NOS3 and GUCY1A1 loci has been reported to be mediated via altered promoter activity, ${ }^{138,141}$ the link between genotype and gene expression at the PDE5A locus is not yet understood.

While the connection between variants involved in nitric oxide signaling and platelet aggregation is obvious, it is still not known how exactly this pathway is involved in plaque formation and atherosclerosis in general. Hints that the pathway might not be only important in atherothrombotic but also preceding processes come from both experimental studies rendering an involvement in the recruitment of inflammatory cells to the vessel wall likely. ${ }^{134,135}$ Furthermore, there is genetic evidence that at least impaired sGC activity might primarily affect atherosclerosis: in a family with high prevalence of premature manifestation of CAD due to a digenic mutation in GUCY1A1 and the gene encoding the chaperone protein CCTeta, four of 11 family members carrying at least one mutation underwent $\mathrm{PCI}$ or coronary artery bypass surgery at a young age but did not suffer from MI. ${ }^{142}$ It will thus be a challenge to identify the underlying cellular and molecular mechanisms to evaluate the potential of modifying this pathway in atherosclerosis. In this respect, cumulative effects of multiple risk alleles which share effects in the NO-cGMP-signaling pathway or respective coexpression networks may be informative. ${ }^{123-125,139,143}$

Despite these data suggesting a role for platelet phenotypes and/or function in atherosclerosis, it has to be mentioned that monogenic diseases influencing platelet phenotypes have not 
been associated with reduced incidence of CVDs. In Glanzmann's thrombasthenia in particular, a disease which is a consequence of deficient $\alpha$ IIb $\beta 3$ integrin function in platelets, cardiovascular events as arterial thrombosis or deep vein thrombosis have been reported (for a review, see Nurden ${ }^{144}$ ). Another example is the rare Bernard-Soulier syndrome that is characterized by defects in the VWF-receptor complex (GPIbV-IX), where also MI has been reported. ${ }^{145}$ However, these reports have to be taken with caution as it is obvious that CVDs and in particular CAD are influenced by several further risk factors that cause the disease despite altered platelet function.

\section{Pharmacological Approaches Targeting Platelets in Atherosclerosis}

Given the findings from basic research, inhibiting platelet function seems like a plausible strategy to prevent atherosclerotic plaque formation and progression. Current platelet treatment targets include COX-1, which mediates AA metabolism, $\mathrm{P}_{2} \mathrm{Y}_{12}$ adenosine diphosphate receptors, and the $\alpha \mathrm{IIb} \beta 3$ glycoprotein receptor. Whereas the latter two are rather targeted/ utilized in specific situations, e.g., after coronary stenting or in acute coronary syndromes, the role of aspirin in both primary and secondary prevention of atherosclerotic disease as well as in animal models has been extensively studied.

In secondary prevention, aspirin has been shown to significantly reduce the incidence of vascular events in patients with acute stroke (absolute reduction: $0.9 \%$ ) or MI (absolute reduction: 3.8\%), previous stroke/transient ischemic attack (absolute reduction: $3.6 \%$ ) or $\mathrm{MI}$ (absolute reduction: 3.5\%), but also other high-risk situations (absolute reduction: $2.1 \%) .{ }^{146}$ These benefits clearly outweigh the risk of bleeding, i.e., the number needed to treat (NNT) to prevent a serious vascular event ranges between 50 and 100 , whereas the number needed to harm ranges between 500 and 1,000 and 5,000 and 10,000 for gastrointestinal bleeding events and hemorrhagic strokes, respectively. ${ }^{147}$ The effect in secondary prevention is thought to be a consequence of preventing atherothrombosis. ${ }^{148}$

In primary prevention of atherosclerotic plaques using aspirin, the situation seems more complex. In animal models, several studies have suggested that AA-related pathways and their inhibition are associated with atherosclerotic plaque formation and/or progression. In $L d l r^{-1-}$ mice fed a Westerntype diet, indomethacin, a nonselective COX inhibitor reduced atherosclerotic plaque formation and reduced expression of, e.g., soluble intercellular adhesion molecule and monocyte chemotactic protein- $1 .^{149}$ That this effect is mediated via COX1 is suggested by the fact that selective inhibition of COX-1 but not COX-2 led to reduced atherosclerotic plaque formation in $A p o E^{-1-}$ mice fed a high-cholesterol diet. ${ }^{150}$ An impact of reduced thromboxane $A_{2}$, the product of COX-1-mediated AA metabolism, on atherosclerotic plaque formation has also been shown by specifically inhibiting its receptor; in this study, however, the unselective inhibition of COX-1 and -2 by indomethacin did not lead to reduced atherosclerotic plaque formation. ${ }^{151}$ Data from human studies do not ultimately clarify the role of aspirin in primary prevention. Although there are early data which suggested a benefit from high-dose aspirin in atherosclerotic plaque progression, ${ }^{152}$ randomized clinical trials in healthy subjects ${ }^{153}$ and meta-analyses ${ }^{154}$ have not been able to show a benefit from aspirin intake that outweighs the increased risk in bleeding. Two recent studies in diabetic or high-risk patients also failed to prove a benefit from aspirin in primary prevention. ${ }^{155,156}$ There may be a subgroup of patients without previous cardiovascular events, but at a risk comparable to that of patients in secondary prevention (predicted $10 \%$ mortality in 10 years) or individuals with a particular genetic background. A very interesting example is the reduction of LDL-cholesterol and cardiovascular events using statins. Here, it has been shown that a high genetic risk score (including 27 variants associated with incident CHD) is associated with a stronger reduction of cardiovascular risk by statin therapy compared with individuals with a low genetic risk score. In an analysis of the JUPITER trial, the NNTs to prevent a cardiovascular event within 10 years were 66,42 , and 25 in the low, intermediate, and high genetic risk groups, respectively. ${ }^{99}$ It is possible that in the sense of precision medicine, individuals could also be identified to specifically benefit from antiplatelet treatment with an unspecific drug such as aspirin if this is identified as the disease-driving pathway. ${ }^{157}$ Also here, knowledge from genome-wide association studies might be useful. Whereas the Womens' and Physician's Health Study did not show a clear benefit from aspirin in primary prevention in the overall study population, ${ }^{153}$ individuals carrying the homozygous GUCY1A3 risk genotype had a benefit from aspirin treatment with a $17 \%$ risk reduction in women and a $51 \%$ reduction in men. In women the NNT treated to avoid one major CVD event was $121{ }^{158}$ Considering higher risk and stronger effects in men, the NNT for GUCY1A3-guided prescription of aspirin could enter the range of clear benefit unless otherwise contraindicated (NNT $\leq 100) .{ }^{159}$ Surprisingly, carriers of the nonrisk allele, either heterozygous or homozygous, did not only lack benefit from aspirin but rather experienced an increased risk compared with placebo. ${ }^{158}$ This is a peculiar observation that remains to be validated and explained. However, it has been shown that the GUCY1A3 genotype is also associated with a response to aspirin therapy with nonrisk allele carriers showing lower on-aspirin platelet reactivity. ${ }^{160}$ One could speculate that in nonrisk allele carriers aspirin shifts platelets toward an increased risk of bleeding which is itself-directly and indirectly via anemia-associated with cardiovascular events. ${ }^{161-163}$ Of note, it also needs to be taken into account that the effect of particular SNPs on CAD risk-and thereby also the presumed effect on the responsiveness to antiplatelet therapies- is rather mediated by altered gene expression than protein function. In contrast to CYP2C19 alleles leading to slower metabolism of clopidogrel to active metabolites and -as a consequence-to increased risk of ischemic events in patients with acute coronary syndromes ${ }^{109,164,165}$, the GUCY1A3 risk allele is associated with reduced expression of the gene. ${ }^{138}$ The influence of reduced sGC protein levels on response to aspirin treatment is, however, thought to be a result of changes in intracellular equilibria: as such, AA influences platelet nitric oxide levels ${ }^{166}$ and GUCY1A3 risk allele carriers presenting reduced sGC protein levels and activity 
might benefit from aspirin to outweigh this effect. ${ }^{160}$ While this remains speculative, such complex interactions need to be taken into account.

\section{Conclusion and Outlook}

In this brief review we have pointed out some recent advances in the understanding how platelets influence atherogenesis, but a comprehensive reporting on all concepts was not within the scope. There is still a large gap to be closed between the clear notion of platelets as inflammatory and atherogenic cellular particles derived from experimental data and prove for this concept in humans. This is based primarily on the fact that platelets have a dual role as drivers of atherosclerosis and executers of arterial thrombosis after plaque rupture. Human data evaluating prognosis originate to a large part from registries, trials, and observational studies that mainly include symptomatic patients after hospitalization and therefore the thrombotic role of platelets in plaque erosion and rupture masks their impact on earlier stages in plaque development. Still, some studies were able to relate platelet phenotypes to CAD by recording $\mathrm{PCI}$ or coronary artery bypass grafting in a nonacute setting. An obstacle that needs to be overcome generally in evaluating atherosclerosis, which is a slowly progressive disease, is the possibility to assess plaque phenotypes in asymptomatic humans over long time ranges. High-resolution imaging such as optical coherence tomography and IVUS are able to characterize coronary plaques, but are invasive techniques precluding a screening of asymptomatic patients. Therefore, a translational realization of all these interesting concepts remains challenging.

\section{Funding}

This study was funded by Deutsche Forschungsgemeinschaft (grants: SFB1123 A2; SFB1123 B2).

\section{Conflict of Interest}

None declared.

\section{References}

1 Nabel EG, Braunwald E. A tale of coronary artery disease and myocardial infarction. N Engl J Med 2012;366(01):54-63

2 Borén J, Williams KJ. The central role of arterial retention of cholesterol-rich apolipoprotein-B-containing lipoproteins in the pathogenesis of atherosclerosis: a triumph of simplicity. Curr Opin Lipidol 2016;27(05):473-483

3 Ridker PM, Everett BM, Thuren T, et al; CANTOS Trial Group. Antiinflammatory therapy with canakinumab for atherosclerotic disease. N Engl J Med 2017;377(12):1119-1131

4 Weber C, von Hundelshausen P. CANTOS trial validates the inflammatory pathogenesis of atherosclerosis: setting the stage for a new chapter in therapeutic targeting. Circ Res 2017;121 (10):1119-1121

5 Tabas I, Lichtman AH. Monocyte-macrophages and T cells in atherosclerosis. Immunity 2017;47(04):621-634

6 Mustard JF, Murphy EA, Rowsell HC, Downie HG. Platelets and atherosclerosis. J Atheroscler Res 1964;4(01):1-28

7 Gawaz M, Langer H, May AE. Platelets in inflammation and atherogenesis. J Clin Invest 2005;115(12):3378-3384
8 Yun KH, Mintz GS, Witzenbichler B, et al. Relationship between platelet reactivity and culprit lesion morphology: an assessment from the ADAPT-DES intravascular ultrasound substudy. JACC Cardiovasc Imaging 2016;9(07):849-854

9 Mangiacapra F, De Bruyne B, Muller O, et al. High residual platelet reactivity after clopidogrel: extent of coronary atherosclerosis and periprocedural myocardial infarction in patients with stable angina undergoing percutaneous coronary intervention. JACC Cardiovasc Interv 2010;3(01):35-40

10 von Hundelshausen P, Weber C. Platelets as immune cells: bridging inflammation and cardiovascular disease. Circ Res 2007;100(01):27-40

11 Karshovska E, Zhao Z, Blanchet X, et al. Hyperreactivity of junctional adhesion molecule A-deficient platelets accelerates atherosclerosis in hyperlipidemic mice. Circ Res 2015;116(04): 587-599

12 Schmitt MM, Megens RT, Zernecke A, et al. Endothelial junctional adhesion molecule-a guides monocytes into flow-dependent predilection sites of atherosclerosis. Circulation 2014;129(01): 66-76

13 Cavusoglu E, Kornecki E, Sobocka MB, et al. Association of plasma levels of F11 receptor/junctional adhesion molecule-A (F11R/JAM-A) with human atherosclerosis. J Am Coll Cardiol 2007; 50(18):1768-1776

14 Karshovska E, Weber C, von Hundelshausen P. Platelet chemokines in health and disease. Thromb Haemost 2013;110(05):894-902

15 Koenen RR, von Hundelshausen P, Nesmelova IV, et al. Disrupting functional interactions between platelet chemokines inhibits atherosclerosis in hyperlipidemic mice. Nat Med 2009;15(01): 97-103

16 von Hundelshausen P, Agten SM, Eckardt V, et al. Chemokine interactome mapping enables tailored intervention in acute and chronic inflammation. Sci Transl Med 2017;9(384):6650

17 Massberg S, Brand K, Grüner S, et al. A critical role of platelet adhesion in the initiation of atherosclerotic lesion formation. J Exp Med 2002;196(07):887-896

18 McCarty OJ, Conley RB, Shentu W, et al. Molecular imaging of activated von Willebrand factor to detect high-risk atherosclerotic phenotype. JACC Cardiovasc Imaging 2010;3(09):947-955

19 McGrath RT, van den Biggelaar M, Byrne B, et al. Altered glycosylation of platelet-derived von Willebrand factor confers resistance to ADAMTS13 proteolysis. Blood 2013;122(25): 4107-4110

20 Doddapattar P, Dhanesha N, Chorawala MR, et al. Endothelial cell-derived von Willebrand factor, but not platelet-derived, promotes atherosclerosis in apolipoprotein E-deficient mice. Arterioscler Thromb Vasc Biol 2018;38(03):520-528

21 Popa M, Tahir S, Elrod J, et al. Role of CD40 and ADAMTS13 in von Willebrand factor-mediated endothelial cell-platelet-monocyte interaction. Proc Natl Acad Sci U S A 2018;115(24):E5556-E5565

22 van Galen KP, Tuinenburg A, Smeets EM, Schutgens RE. Von Willebrand factor deficiency and atherosclerosis. Blood Rev 2012;26(05):189-196

23 Holm E, Osooli M, Steen Carlsson K, Berntorp E. Cardiovascular disease-related hospitalization and mortality among persons with von Willebrand disease: a nationwide register study in Sweden. Haemophilia 2019;25(01):109-115

24 Huo Y, Schober A, Forlow SB, et al. Circulating activated platelets exacerbate atherosclerosis in mice deficient in apolipoprotein $\mathrm{E}$. Nat Med 2003;9(01):61-67

25 Burger PC, Wagner DD. Platelet P-selectin facilitates atherosclerotic lesion development. Blood 2003;101(07):2661-2666

26 Schmitt C, Abt M, Ciorciaro C, et al. First-in-man study with inclacumab, a human monoclonal antibody against P-selectin. J Cardiovasc Pharmacol 2015;65(06):611-619

27 Ataga KI, Kutlar A, Kanter J, et al. Crizanlizumab for the prevention of pain crises in sickle cell disease. N Engl J Med 2017;376 (05):429-439 
28 Gawaz M. Novel ligands for platelet glycoprotein VI. Thromb Haemost 2018;118(03):435-436

29 Bültmann A, Li Z, Wagner S, et al. Impact of glycoprotein VI and platelet adhesion on atherosclerosis-a possible role of fibronectin. J Mol Cell Cardiol 2010;49(03):532-542

30 Metzger K, Vogel S, Chatterjee M, et al. High-frequency ultrasound-guided disruption of glycoprotein VI-targeted microbubbles targets atheroprogressison in mice. Biomaterials 2015; 36:80-89

31 Rayes J, Watson SP, Nieswandt B. Functional significance of the platelet immune receptors GPVI and CLEC-2. J Clin Invest 2019; 129(01):12-23

32 Jamasbi J, Megens RT, Bianchini M, et al. Differential inhibition of human atherosclerotic plaque-induced platelet activation by dimeric GPVI-Fc and anti-GPVI antibodies: functional and imaging studies. J Am Coll Cardiol 2015;65(22):2404-2415

33 Schulz C, Penz S, Hoffmann C, et al. Platelet GPVI binds to collagenous structures in the core region of human atheromatous plaque and is critical for atheroprogression in vivo. Basic Res Cardiol 2008;103(04):356-367

34 Ungerer M, Rosport K, Bültmann A, et al. Novel antiplatelet drug revacept (dimeric glycoprotein $\mathrm{VI}-\mathrm{Fc}$ ) specifically and efficiently inhibited collagen-induced platelet aggregation without affecting general hemostasis in humans. Circulation 2011;123(17): 1891-1899

35 Schüpke S, Hein-Rothweiler R, Mayer K, et al; ISAR-PLASTERTrial Investigators. Revacept, a novel inhibitor of platelet adhesion, in patients undergoing elective PCI-design and rationale of the randomized ISAR-PLASTER trial. Thromb Haemost 2019;119 (09):1539-1545

36 Goldmann L, Duan R, Kragh T, et al. Oral Bruton tyrosine kinase inhibitors block activation of the platelet Fc receptor CD32a (FcrRIIA): a new option in HIT? Blood Adv 2019;3(23):4021-4033

37 Busygina K, Jamasbi J, Seiler T, et al. Oral Bruton tyrosine kinase inhibitors selectively block atherosclerotic plaque-triggered thrombus formation in humans. Blood 2018;131(24): 2605-2616

38 Busygina K, Denzinger V, Bernlochner I, Weber C, Lorenz R, Siess W. Btk inhibitors as first oral atherothrombosis-selective antiplatelet drugs? Thromb Haemost 2019;119(08):1212-1221

39 Soehnlein O. Decision shaping neutrophil-platelet interplay in inflammation: from physiology to intervention. Eur J Clin Invest 2018;48(02):e12871

40 Brinkmann V, Reichard U, Goosmann C, et al. Neutrophil extracellular traps kill bacteria. Science 2004;303(5663):1532-1535

41 Wang Y, Li M, Stadler S, et al. Histone hypercitrullination mediates chromatin decondensation and neutrophil extracellular trap formation. J Cell Biol 2009;184(02):205-213

42 Megens RT, Vijayan S, Lievens D, et al. Presence of luminal neutrophil extracellular traps in atherosclerosis. Thromb Haemost 2012;107(03):597-598

43 Warnatsch A, Ioannou M, Wang Q Papayannopoulos V. Inflammation. Neutrophil extracellular traps license macrophages for cytokine production in atherosclerosis. Science 2015;349 (6245):316-320

44 Knight JS, Luo W, O'Dell AA, et al. Peptidylarginine deiminase inhibition reduces vascular damage and modulates innate immune responses in murine models of atherosclerosis. Circ Res 2014;114(06):947-956

45 Döring Y, Soehnlein O, Weber C. Neutrophil extracellular traps in atherosclerosis and atherothrombosis. Circ Res 2017;120(04): 736-743

46 Silvestre-Roig C, Braster Q, Wichapong K, et al. Externalized histone $\mathrm{H} 4$ orchestrates chronic inflammation by inducing lytic cell death. Nature 2019;569(7755):236-240

47 Clark SR, Ma AC, Tavener SA, et al. Platelet TLR4 activates neutrophil extracellular traps to ensnare bacteria in septic blood. Nat Med 2007;13(04):463-469
48 Caudrillier A, Kessenbrock K, Gilliss BM, et al. Platelets induce neutrophil extracellular traps in transfusion-related acute lung injury. J Clin Invest 2012;122(07):2661-2671

49 Maugeri N, Capobianco A, Rovere-Querini P, et al. Platelet microparticles sustain autophagy-associated activation of neutrophils in systemic sclerosis. Sci Transl Med 2018;10(451):10

50 Maugeri N, Campana L, Gavina M, et al. Activated platelets present high mobility group box 1 to neutrophils, inducing autophagy and promoting the extrusion of neutrophil extracellular traps. J Thromb Haemost 2014;12(12):2074-2088

51 Rossaint J, Herter JM, Van Aken H, et al. Synchronized integrin engagement and chemokine activation is crucial in neutrophil extracellular trap-mediated sterile inflammation. Blood 2014; 123(16):2573-2584

52 Grommes J, Alard JE, Drechsler M, et al. Disruption of plateletderived chemokine heteromers prevents neutrophil extravasation in acute lung injury. Am J Respir Crit Care Med 2012;185 (06):628-636

53 Elaskalani O, Abdol Razak NB, Metharom P. Neutrophil extracellular traps induce aggregation of washed human platelets independently of extracellular DNA and histones. Cell Commun Signal 2018;16(01):24

54 Ramirez GA, Manfredi AA, Maugeri N. Misunderstandings between platelets and neutrophils build in chronic inflammation. Front Immunol 2019;10:2491

55 Fahy E, Cotter D, Sud M, Subramaniam S. Lipid classification, structures and tools. Biochim Biophys Acta 2011;1811(11): 637-647

56 Slatter DA, Aldrovandi M, O'Connor A, et al. Mapping the human platelet lipidome reveals cytosolic phospholipase A2 as a regulator of mitochondrial bioenergetics during activation. Cell Metab 2016;23(05):930-944

57 O'Donnell VB, Murphy RC, Watson SP. Platelet lipidomics: modern day perspective on lipid discovery and characterization in platelets. Circ Res 2014;114(07):1185-1203

58 McFadyen JD, Peter K. Platelet lipidomics and function: joining the dots. Blood 2018;132(05):465-466

59 Chatterjee M. Platelet lipidome: dismantling the "Trojan horse" in the bloodstream. J Thromb Haemost 2020;18(03):543-557

60 Mason RP, Libby P, Bhatt DL. Emerging mechanisms of cardiovascular protection for the omega-3 fatty acid eicosapentaenoic acid. Arterioscler Thromb Vasc Biol 2020;40(05):1135-1147

61 Bhatt DL, Steg PG, Miller M, et al; REDUCE-IT Investigators. Cardiovascular risk reduction with icosapent ethyl for hypertriglyceridemia. N Engl J Med 2019;380(01):11-22

62 Yokoyama M, Origasa H, Matsuzaki M, et al; Japan EPA lipid intervention study (JELIS) Investigators. Effects of eicosapentaenoic acid on major coronary events in hypercholesterolaemic patients (JELIS): a randomised open-label, blinded endpoint analysis. Lancet 2007;369(9567):1090-1098

63 Sato M, Katsuki Y, Fukuhara K, et al. Effects of highly purified ethyl all-cis-5,8,11,14,17-icosapentaenoate (EPA-E) on rabbit platelets. Biol Pharm Bull 1993;16(04):362-367

64 Kobayashi T, Tahara Y, Matsumoto M, et al. Roles of thromboxane $\mathrm{A}(2)$ and prostacyclin in the development of atherosclerosis in apoE-deficient mice. J Clin Invest 2004;114(06):784-794

65 Ricciotti E, FitzGerald GA. Prostaglandins and inflammation. Arterioscler Thromb Vasc Biol 2011;31(05):986-1000

66 Egan KM, Lawson JA, Fries S, et al. COX-2-derived prostacyclin confers atheroprotection on female mice. Science 2004;306 (5703):1954-1957

67 Feldman M, Jialal I, Devaraj S, Cryer B. Effects of low-dose aspirin on serum C-reactive protein and thromboxane B2 concentrations: a placebo-controlled study using a highly sensitive Creactive protein assay. J Am Coll Cardiol 2001;37(08):2036-2041

68 McNeil JJ, Wolfe R, Woods RL, et al; ASPREE Investigator Group. Effect of aspirin on cardiovascular events and bleeding in the healthy elderly. N Engl J Med 2018;379(16):1509-1518 
69 Maceyka M, Spiegel S. Sphingolipid metabolites in inflammatory disease. Nature 2014;510(7503):58-67

70 Yu Z, Peng Q, Huang Y. Potential therapeutic targets for atherosclerosis in sphingolipid metabolism. Clin Sci (Lond) 2019;133 (06):763-776

71 Smyth SS, Mueller P, Yang F, Brandon JA, Morris AJ. Arguing the case for the autotaxin-lysophosphatidic acid-lipid phosphate phosphatase 3-signaling nexus in the development and complications of atherosclerosis. Arterioscler Thromb Vasc Biol 2014; 34(03):479-486

72 Edsfeldt A, Dunér P, Ståhlman M, et al. Sphingolipids contribute to human atherosclerotic plaque inflammation. Arterioscler Thromb Vasc Biol 2016;36(06):1132-1140

73 Jonnalagadda D, Sunkara M, Morris AJ, Whiteheart SW. Granulemediated release of sphingosine-1-phosphate by activated platelets. Biochim Biophys Acta 2014;1841(11):1581-1589

74 Yatomi Y, Ruan F, Hakomori S, Igarashi Y. Sphingosine-1-phosphate: a platelet-activating sphingolipid released from agoniststimulated human platelets. Blood 1995;86(01):193-202

75 Zhang L, Orban M, Lorenz M, et al. A novel role of sphingosine 1phosphate receptor S1 pr1 in mouse thrombopoiesis. J Exp Med 2012;209(12):2165-2181

76 English D, Welch Z, Kovala AT, et al. Sphingosine 1-phosphate released from platelets during clotting accounts for the potent endothelial cell chemotactic activity of blood serum and provides a novel link between hemostasis and angiogenesis. FASEB J 2000;14(14):2255-2265

77 Bodin S, Tronchère H, Payrastre B. Lipid rafts are critical membrane domains in blood platelet activation processes. Biochim Biophys Acta 2003;1610(02):247-257

78 Yang H, Kim A, David T, et al. TMEM16F forms a Ca2+-activated cation channel required for lipid scrambling in platelets during blood coagulation. Cell 2012;151(01):111-122

79 Chatterjee M, Rath D, Schlotterbeck J, et al. Regulation of oxidized platelet lipidome: implications for coronary artery disease. Eur Heart J 2017;38(25):1993-2005

80 Diehl P, Nienaber F, Zaldivia MTK, et al. Lysophosphatidylcholine is a major component of platelet microvesicles promoting platelet activation and reporting atherosclerotic plaque instability. Thromb Haemost 2019;119(08):1295-1310

81 Vajen T, Mause SF, Koenen RR. Microvesicles from platelets: novel drivers of vascular inflammation. Thromb Haemost 2015;114 (02):228-236

82 Bolen AL, Naren AP, Yarlagadda S, et al. The phospholipase A1 activity of lysophospholipase A-I links platelet activation to LPA production during blood coagulation. J Lipid Res 2011;52(05): 958-970

83 Siess W, Zangl KJ, Essler M, et al. Lysophosphatidic acid mediates the rapid activation of platelets and endothelial cells by mildly oxidized low density lipoprotein and accumulates in human atherosclerotic lesions. Proc Natl Acad Sci U S A 1999;96(12): 6931-6936

84 Fessler MB, Rose K, Zhang Y, Jaramillo R, Zeldin DC. Relationship between serum cholesterol and indices of erythrocytes and platelets in the US population. J Lipid Res 2013;54(11):3177-3188

85 Barrett TJ, Schlegel M, Zhou F, et al. Platelet regulation of myeloid suppressor of cytokine signaling 3 accelerates atherosclerosis. Sci Transl Med 2019;11(517):481

86 Wang N, Tall AR. Cholesterol in platelet biogenesis and activation. Blood 2016;127(16):1949-1953

87 Ahmadsei M, Lievens D, Weber C, von Hundelshausen P, Gerdes $\mathrm{N}$. Immune-mediated and lipid-mediated platelet function in atherosclerosis. Curr Opin Lipidol 2015;26(05):438-448

88 Glynn RJ, Danielson E, Fonseca FA, et al. A randomized trial of rosuvastatin in the prevention of venous thromboembolism. N Engl J Med 2009;360(18):1851-1861

89 Oesterle A, Laufs U, Liao JK. Pleiotropic effects of statins on the cardiovascular system. Circ Res 2017;120(01):229-243
90 Aslan JE, McCarty OJ. Rho GTPases in platelet function. J Thromb Haemost 2013;11(01):35-46

91 Podrez EA, Byzova TV, Febbraio M, et al. Platelet CD36 links hyperlipidemia, oxidant stress and a prothrombotic phenotype. Nat Med 2007;13(09):1086-1095

92 Badrnya S, Schrottmaier WC, Kral JB, et al. Platelets mediate oxidized low-density lipoprotein-induced monocyte extravasation and foam cell formation. Arterioscler Thromb Vasc Biol 2014;34(03):571-580

93 Barale C, Bonomo K, Frascaroli C, et al. Platelet function and activation markers in primary hypercholesterolemia treated with anti-PCSK9 monoclonal antibody: A 12-month follow-up. Nutr Metab Cardiovasc Dis 2020;30(02):282-291

94 Navarese EP, Kolodziejczak M, Winter MP, et al. Association of PCSK9 with platelet reactivity in patients with acute coronary syndrome treated with prasugrel or ticagrelor: The PCSK9REACT study. Int J Cardiol 2017;227:644-649

95 Chu SG, Becker RC, Berger PB, et al. Mean platelet volume as a predictor of cardiovascular risk: a systematic review and metaanalysis. J Thromb Haemost 2010;8(01):148-156

96 Boos CJ, Lip GY. Assessment of mean platelet volume in coronary artery disease - what does it mean? Thromb Res 2007;120(01): $11-13$

97 Lancé MD, Sloep M, Henskens YM, Marcus MA. Mean platelet volume as a diagnostic marker for cardiovascular disease: drawbacks of preanalytical conditions and measuring techniques. Clin Appl Thromb Hemost 2012;18(06):561-568

98 Soranzo N, Spector TD, Mangino M, et al. A genome-wide metaanalysis identifies 22 loci associated with eight hematological parameters in the HaemGen consortium. Nat Genet 2009;41 (11):1182-1190

99 Mega JL, Stitziel NO, Smith JG, et al. Genetic risk, coronary heart disease events, and the clinical benefit of statin therapy: an analysis of primary and secondary prevention trials. Lancet 2015;385(9984):2264-2271

100 Iotchkova V, Huang J, Morris JA, et al; UK10K Consortium. Discovery and refinement of genetic loci associated with cardiometabolic risk using dense imputation maps. Nat Genet 2016; 48(11):1303-1312

101 Astle WJ, Elding H, Jiang T, et al. The allelic landscape of human blood cell trait variation and links to common complex disease. Cell 2016;167(05):1415-1429.e19

102 Kamatani Y, Matsuda K, Okada Y, et al. Genome-wide association study of hematological and biochemical traits in a Japanese population. Nat Genet 2010;42(03):210-215

103 Gieger C, Radhakrishnan A, Cvejic A, et al. New gene functions in megakaryopoiesis and platelet formation. Nature 2011;480 (7376):201-208

104 Qayyum R, Snively BM, Ziv E, et al. A meta-analysis and genomewide association study of platelet count and mean platelet volume in African Americans. PLoS Genet 2012;8(03):e1002491

$105 \mathrm{Li}$ J, Glessner JT, Zhang H, et al. GWAS of blood cell traits identifies novel associated loci and epistatic interactions in Caucasian and African-American children. Hum Mol Genet 2013;22(07): 1457-1464

106 Shameer K, Denny JC, Ding K, et al. A genome- and phenomewide association study to identify genetic variants influencing platelet count and volume and their pleiotropic effects. Hum Genet 2014;133(01):95-109

107 Schick UM, Jain D, Hodonsky CJ, et al. Genome-wide association study of platelet count identifies ancestry-specific loci in Hispanic/Latino Americans. Am J Hum Genet 2016;98(02): 229-242

108 Kanai M, Akiyama M, Takahashi A, et al. Genetic analysis of quantitative traits in the Japanese population links cell types to complex human diseases. Nat Genet 2018;50(03):390-400

109 Shuldiner AR, O'Connell JR, Bliden KP, et al. Association of cytochrome P450 2C19 genotype with the antiplatelet effect 
and clinical efficacy of clopidogrel therapy. JAMA 2009;302(08): 849-857

110 Mega JL, Close SL, Wiviott SD, et al. Cytochrome p-450 polymorphisms and response to clopidogrel. N Engl J Med 2009;360 (04):354-362

111 Johnson AD, Yanek LR, Chen MH, et al. Genome-wide metaanalyses identifies seven loci associated with platelet aggregation in response to agonists. Nat Genet 2010;42(07):608-613

112 Erdmann J, Kessler T, Munoz Venegas L, Schunkert H. A decade of genome-wide association studies for coronary artery disease: the challenges ahead. Cardiovasc Res 2018;114(09):1241-1257

113 Barrett JC, Clayton DG, Concannon P, et al; Type 1 Diabetes Genetics Consortium. Genome-wide association study and meta-analysis find that over 40 loci affect risk of type 1 diabetes. Nat Genet 2009;41(06):703-707

114 Ehret GB, Munroe PB, Rice KM, et al; International Consortium for Blood Pressure Genome-Wide Association Studies; CARDIoGRAM consortium; CKDGen Consortium; KidneyGen Consortium; EchoGen consortium; CHARGE-HF consortium. Genetic variants in novel pathways influence blood pressure and cardiovascular disease risk. Nature 2011;478(7367):103-109

115 Wain LV, Verwoert GC, O'Reilly PF, et al; LifeLines Cohort Study; EchoGen consortium; AortaGen Consortium; CHARGE Consortium Heart Failure Working Group; KidneyGen consortium; CKDGen consortium; Cardiogenics consortium; CardioGram. Genome-wide association study identifies six new loci influencing pulse pressure and mean arterial pressure. Nat Genet 2011; 43(10):1005-1011

116 Hunt KA, Zhernakova A, Turner G, et al. Newly identified genetic risk variants for celiac disease related to the immune response. Nat Genet 2008;40(04):395-402

117 Tong W, Lodish HF. Lnk inhibits Tpo-mpl signaling and Tpo-mediated megakaryocytopoiesis. J Exp Med 2004;200(05):569-580

118 Bersenev A, Wu C, Balcerek J, Tong W. Lnk controls mouse hematopoietic stem cell self-renewal and quiescence through direct interactions with JAK2. J Clin Invest 2008;118(08): 2832-2844

119 Takizawa H, Nishimura S, Takayama N, et al. Lnk regulates integrin alphallbbeta3 outside-in signaling in mouse platelets, leading to stabilization of thrombus development in vivo. J Clin Invest 2010;120(01):179-190

120 Christiansen MK, Larsen SB, Nyegaard M, et al. The SH2B3 and KCNK5 loci may be implicated in regulation of platelet count, volume, and maturity. Thromb Res 2017;158:86-92

121 Wang W, Tang Y, Wang Y, et al. LNK/SH2B3 loss of function promotes atherosclerosis and thrombosis. Circ Res 2016;119 (06):e91-e103

122 Wobst J, Schunkert H, Kessler T. Genetic alterations in the NOcGMP pathway and cardiovascular risk. Nitric Oxide 2018; 76:105-112

123 Nikpay M, Goel A, Won HH, et al. A comprehensive 1,000 Genomes-based genome-wide association meta-analysis of coronary artery disease. Nat Genet 2015;47(10):1121-1130

124 Deloukas P, Kanoni S, Willenborg C, et al; CARDIoGRAMplusC4D Consortium; DIAGRAM Consortium; CARDIOGENICS Consortium; MuTHER Consortium; Wellcome Trust Case Control Consortium. Large-scale association analysis identifies new risk loci for coronary artery disease. Nat Genet 2013;45(01):25-33

125 Webb TR, Erdmann J, Stirrups KE, et al; Wellcome Trust Case Control Consortium; MORGAM Investigators; Myocardial Infarction Genetics and CARDIoGRAM Exome Consortia Investigators. Systematic evaluation of pleiotropy identifies 6 further loci associated with coronary artery disease. J Am Coll Cardiol 2017;69(07):823-836

126 Klarin D, Zhu QM, Emdin CA, et al; CARDIoGRAMplusC4D Consortium. Genetic analysis in UK Biobank links insulin resistance and transendothelial migration pathways to coronary artery disease. Nat Genet 2017;49(09):1392-1397
127 Schunkert H, König IR, Kathiresan S, et al; Cardiogenics; CARDIoGRAM Consortium. Large-scale association analysis identifies 13 new susceptibility loci for coronary artery disease. Nat Genet 2011;43(04):333-338

128 Kathiresan S, Voight BF, Purcell S, et al; Myocardial Infarction Genetics Consortium; Wellcome Trust Case Control Consortium. Genome-wide association of early-onset myocardial infarction with single nucleotide polymorphisms and copy number variants. Nat Genet 2009;41(03):334-341

129 Ignarro LJ, Buga GM, Wood KS, Byrns RE, Chaudhuri G. Endothelium-derived relaxing factor produced and released from artery and vein is nitric oxide. Proc Natl Acad Sci US A 1987;84(24):9265-9269

130 Mellion BT, Ignarro LJ, Ohlstein EH, Pontecorvo EG, Hyman AL, Kadowitz PJ. Evidence for the inhibitory role of guanosine 3', 5'monophosphate in ADP-induced human platelet aggregation in the presence of nitric oxide and related vasodilators. Blood 1981; 57(05):946-955

131 Moro MA, Russel RJ, Cellek S, et al. cGMP mediates the vascular and platelet actions of nitric oxide: confirmation using an inhibitor of the soluble guanylyl cyclase. Proc Natl Acad Sci U S A 1996;93(04):1480-1485

132 Bekendam RH, Iyu D, Passam F, et al. Protein disulfide isomerase regulation by nitric oxide maintains vascular quiescence and controls thrombus formation. J Thromb Haemost 2018;16(11): 2322-2335

133 Lukowski R, Weinmeister P, Bernhard D, et al. Role of smooth muscle cGMP/cGKI signaling in murine vascular restenosis. Arterioscler Thromb Vasc Biol 2008;28(07):1244-1250

134 Melichar VO, Behr-Roussel D, Zabel U, et al. Reduced cGMP signaling associated with neointimal proliferation and vascular dysfunction in late-stage atherosclerosis. Proc Natl Acad Sci U S A 2004;101(47):16671-16676

135 Ahluwalia A, Foster P, Scotland RS, et al. Antiinflammatory activity of soluble guanylate cyclase: cGMP-dependent downregulation of P-selectin expression and leukocyte recruitment. Proc Natl Acad Sci U S A 2004;101(05):1386-1391

136 Salvi E, Kutalik Z, Glorioso N, et al. Genomewide association study using a high-density single nucleotide polymorphism array and case-control design identifies a novel essential hypertension susceptibility locus in the promoter region of endothelial NO synthase. Hypertension 2012;59(02):248-255

137 Emdin CA, Khera AV, Klarin D, et al. Phenotypic consequences of a genetic predisposition to enhanced nitric oxide signaling. Circulation 2018;137(03):222-232

138 Kessler T, Wobst J, Wolf B, et al. Functional characterization of the GUCY1A3 coronary artery disease risk locus. Circulation 2017;136(05):476-489

139 Nelson CP, Goel A, Butterworth AS, et al; EPIC-CVD Consortium; CARDIoGRAMplusC4D; UK Biobank CardioMetabolic Consortium CHD working group. Association analyses based on false discovery rate implicate new loci for coronary artery disease. Nat Genet 2017;49(09):1385-1391

140 Franzén O, Ermel R, Cohain A, et al. Cardiometabolic risk loci share downstream cis- and trans-gene regulation across tissues and diseases. Science 2016;353(6301):827-830

141 Salvi E, Kuznetsova T, Thijs L, et al. Target sequencing, cell experiments, and a population study establish endothelial nitric oxide synthase (eNOS) gene as hypertension susceptibility gene. Hypertension 2013;62(05):844-852

142 Erdmann J, Stark K, Esslinger UB, et al; CARDIoGRAM. Dysfunctional nitric oxide signalling increases risk of myocardial infarction. Nature 2013;504(7480):432-436

143 Zeng L, Talukdar HA, Koplev S, et al. Contribution of gene regulatory networks to heritability of coronary artery disease. J Am Coll Cardiol 2019;73(23):2946-2957

144 Nurden AT. Should studies on Glanzmann thrombasthenia not be telling us more about cardiovascular disease and other major illnesses? Blood Rev 2017;31(05):287-299 
145 Girolami A, Vettore S, Vianello F, Berti de Marinis G, Fabris F. Myocardial infarction in two cousins heterozygous for ASN41HIS autosomal dominant variant of Bernard-Soulier syndrome. J Thromb Thrombolysis 2012;34(04):513-517

146 Collaboration AT; Antithrombotic Trialists' Collaboration. Collaborative meta-analysis of randomised trials of antiplatelet therapy for prevention of death, myocardial infarction, and stroke in high risk patients. BMJ 2002;324(7329):71-86

147 Patrono C, García Rodríguez LA, Landolfi R, Baigent C. Low-dose aspirin for the prevention of atherothrombosis. N Engl J Med 2005;353(22):2373-2383

148 Davì G, Patrono C. Platelet activation and atherothrombosis. N Engl J Med 2007;357(24):2482-2494

149 Praticò D, Tillmann C, Zhang ZB, Li H, FitzGerald GA. Acceleration of atherogenesis by COX-1-dependent prostanoid formation in low density lipoprotein receptor knockout mice. Proc Natl Acad Sci U S A 2001;98(06):3358-3363

150 Belton OA, Duffy A, Toomey S, Fitzgerald DJ. Cyclooxygenase isoforms and platelet vessel wall interactions in the apolipoprotein E knockout mouse model of atherosclerosis. Circulation 2003;108(24):3017-3023

151 Egan KM, Wang M, Fries S, et al. Cyclooxygenases, thromboxane, and atherosclerosis: plaque destabilization by cyclooxygenase- 2 inhibition combined with thromboxane receptor antagonism. Circulation 2005;111(03):334-342

152 Ranke C, Hecker H, Creutzig A, Alexander K. Dose-dependent effect of aspirin on carotid atherosclerosis. Circulation 1993;87 (06):1873-1879

153 Ridker PM, Cook NR, Lee IM, et al. A randomized trial of low-dose aspirin in the primary prevention of cardiovascular disease in women. N Engl J Med 2005;352(13):1293-1304

154 Baigent C, Blackwell L, Collins R, et al; Antithrombotic Trialists' (ATT) Collaboration. Aspirin in the primary and secondary prevention of vascular disease: collaborative meta-analysis of individual participant data from randomised trials. Lancet 2009;373 (9678):1849-1860

155 Bowman L, Mafham M, Wallendszus K, et al; ASCEND Study Collaborative Group. Effects of aspirin for primary prevention in persons with diabetes mellitus. N Engl J Med 2018;379(16):1529-1539
156 Gaziano JM, Brotons C, Coppolecchia R, et al; ARRIVE Executive Committee. Use of aspirin to reduce risk of initial vascular events in patients at moderate risk of cardiovascular disease (ARRIVE): a randomised, double-blind, placebo-controlled trial. Lancet 2018;392(10152):1036-1046

157 Khera AV, Kathiresan S. Is coronary atherosclerosis one disease or many? Setting realistic expectations for precision medicine. Circulation 2017;135(11):1005-1007

158 Hall KT, Kessler T, Buring JE, et al. Genetic variation at the coronary artery disease risk locus GUCY1A3 modifies cardiovascular disease prevention effects of aspirin. Eur Heart J 2019;40 (41):3385-3392

159 Patrono $C$. The multifaceted clinical readouts of platelet inhibition by low-dose aspirin. J Am Coll Cardiol 2015;66(01):74-85

160 Kessler T, Wolf B, Eriksson N, et al. Association of the coronary artery disease risk gene GUCY1A3 with ischaemic events after coronary intervention. Cardiovasc Res 2019;115(10):1512-1518

161 Seaman CD, George KM, Ragni M, Folsom AR. Association of von Willebrand factor deficiency with prevalent cardiovascular disease and asymptomatic carotid atherosclerosis: The Atherosclerosis Risk in Communities Study. Thromb Res 2016;144:236-238

162 Eikelboom JW, Mehta SR, Anand SS, Xie C, Fox KA, Yusuf S. Adverse impact of bleeding on prognosis in patients with acute coronary syndromes. Circulation 2006;114(08):774-782

163 Westenbrink BD, Alings M, Connolly SJ, et al. Anemia predicts thromboembolic events, bleeding complications and mortality in patients with atrial fibrillation: insights from the RE-LY trial. J Thromb Haemost 2015;13(05):699-707

164 Simon T, Verstuyft C, Mary-Krause M, et al; French Registry of Acute ST-Elevation and Non-ST-Elevation Myocardial Infarction (FAST-MI) Investigators. Genetic determinants of response to clopidogrel and cardiovascular events. N Engl J Med 2009;360 (04):363-375

165 Hulot JS, Bura A, Villard E, et al. Cytochrome P450 2C19 loss-offunction polymorphism is a major determinant of clopidogrel responsiveness in healthy subjects. Blood 2006;108(07):2244-2247

166 Signorello MG, Segantin A, Leoncini G. The arachidonic acid effect on platelet nitric oxide level. Biochim Biophys Acta 2009;1791(11):1084-1092 